\title{
Incisões relaxantes limbares durante a cirurgia de catarata: resultados após seguimento de um ano
}

\author{
Limbal relaxing incisions during cataract surgery:one-year follow-up
}

\author{
João Carlos Arraes ${ }^{1}$ \\ FernandoCunha ${ }^{2}$ \\ Tatiana Azevedo Arraes $^{3}$ \\ Ronald Cavalvanti ${ }^{4}$ \\ Marcelo Ventura ${ }^{5}$
}

\section{RESUMO}

Objetivo: Avaliar a variação do astigmatismo entre o pré-operatório, $1^{\circ}$ mês e $12^{\circ}$ mês pós-operatórios dos pacientes submetidos à cirurgia de catarata, com realização de incisões relaxantes limbares para redução do astigmatismo pré-operatório. Métodos: Foram avaliados 16 pacientes submetidos a cirurgia de catarata pela técnica de facoemulsificação através de incisão escleral tunelizada de $5,5 \mathrm{~mm}$, na Fundação Altino Ventura, no período entre abril e julho de 2002, na qual foram realizados incisões relaxantes no limbo (IRL), seguindo o nomograma modificado de Gills (1D-1 IRL de 6 mm; 1-2D-2 IRL de 6 mm;2-3D-2 IRL de 8 mm), nos meridianos corneanos mais curvos determinados por topografia corneana pré-operatória. Resultados: Ocorreu redução significante do astigmatismo pré-operatório no $1^{\circ}$ mês pós-operatório, no grupo de 2 incisões relaxantes no limbo de $6 \mathrm{~mm}$ ( $57 \%$ do astigmatismo topográfico e $87 \%$ do refracional) e o de 2 incisões relaxantes no limbo de $8 \mathrm{~mm}$ ( $50 \%$ do astigmatismo topográfico e $65 \%$ do refracional), mantendo-se sem alteração significante este astigmatismo até o $12^{\circ}$ mês pós-operatório. $\mathrm{O}$ grupo de 1 incisão relaxante no limbo de 6 mm não alcançou redução significante do astigmatismo, no entanto, não houve alteração significante até o $12^{\circ}$ mês pós-operatório. Não foram observadas, ainda, complicações pós-operatórias como "glare", aniseiconia, diplopia, desconforto, infecção da ferida e afinamento ou ectasia corneana. Conclusão: A realização de 2 incisões relaxantes no limbo de 8 e $6 \mathrm{~mm}$, para correção de astigmatismo pré-operatório de 2 a 3 e 1 a 2 dioptrias, respectivamente, mostraram-se eficazes, seguras e com efeito estável ao longo do primeiro ano de acompanhamento pós-operatório. A realização de 1 incisão relaxante no limbo de $6 \mathrm{~mm}$ para redução de 1 dioptria de astigmatismo pré-operatório não se mostrou eficaz, no entanto, não levou a complicações pós-operatórias significativas.

Descritores: Astigmatismo/cirurgia; Extração de catarata; Limbo da córnea/cirurgia; Topografia da córnea; Cuidados pré-operatórios; Cuidados pós-operatórios; Estudo comparativo; Seguimentos

\section{INTRODUÇÃO}

A freqüência significante de astigmatismo na população, fez necessário o surgimento de métodos para sua correção durante a cirurgia da catarata, melhorando, assim, a acuidade visual pós-operatória não-corrigida ${ }^{(1-5)}$. Esses métodos devem, no entanto, preservar a asfericidade da córnea ${ }^{(6)}$.

As incisões relaxantes limbares mostram-se eficazes na redução do astigmatismo pré-operatório entre 1 e 3 dioptrias, não apresentam complicações durante a sua realização e no pós-operatório precoce ${ }^{(7-10)}$. 
O efeito de incisões relaxantes arqueadas e radiais na córnea podem variar com o tempo ${ }^{(11)}$. Estas quando realizadas no limbo, por serem mais distantes do centro óptico da córnea, podem ser mais estáveis com o tempo e apresentarem menos complicações $^{(7)}$.

O objetivo do presente estudo foi, portanto, avaliar a variação do astigmatismo refracional e topográfico entre o préoperatório, $1^{\circ}$ mês pós-operatório e $12^{\circ}$ mês pós-operatório dos pacientes submetidos a facoemulsificação e implante de lente intra-ocular, com realização de incisões relaxantes limbares para redução do astigmatismo pré-operatório no período entre abril e julho de 2002.

\section{MÉTODOS}

Após aprovação pelo comitê de ética e pesquisa da Fundação Altino Ventura e assinatura de um Termo de Consentimento Livre e Esclarecido, foram incluídos no estudo 16 pacientes.

Foram avaliados os pacientes submetidos a facoemulsificação e implante de lente intra-ocular com realização de incisões relaxantes limbares para redução do astigmatismo pré-operatório entre abril e julho de 2002. Foram realizados, nestes pacientes, consulta oftalmológica completa e topografia corneana computadorizada. Foram analisados os dados referentes ao momento pré-operatório, $1^{\circ}$ e $12^{\circ}$ mês pós-operatório do astigmatismo refracional e topográfico, da alteração dos vetores ceratométricos e refratométricos utilizando a fórmula de Holladay, da acuidade visual pós-operatória e dos sinais e sintomas de complicações pós-operatórias, como diplopia, aniseiconia, afinamentos e ectasias corneanas, desconforto e ofuscamento.

Os resultados de variáveis contínuas foram expressos por suas médias aritméticas e respectivos desvios-padrão, ou valores medianos e respectivas distâncias inter-quartil. Foi utilizado o teste t-Student para amostras pareadas na avaliação de possíveis diferenças entre médias. O teste de Wilcoxon foi usado para verificar possíveis diferenças entre medianas. $\mathrm{p}<0,05$ foi utilizado para rejeitar a hipótese de nulidade.

\section{RESULTADOS}

O valor mediano da acuidade visual corrigida pré-operatória foi de 0,05 (movimento de mãos a 0,2 ), atingindo $0,8(0,25$ a 1) no $12^{\circ}$ mês pós-operatório ( $\left.\mathrm{p}<0,0001\right)$. Os dados do astigmatismo refracional e topográfico do pré-operatório, $1^{\circ}$ mês e $12^{\circ}$ mês pós-operatórios estão descritos na tabela 1 .

As incisões relaxantes limbares levaram a uma redução significante do valor médio do astigmatismo pré-operatório tanto refracional (64\%) quanto topográfico (45\%) já no primeiro mês pós-operatório. Esta redução manteve-se, sem apresentar diferença significante dos valores dos astigmatismos entre o $1^{\circ}$ e $12^{\circ}$ mês pós-operatório (Gráfico 1 ).

Redução significante foi observada no $1^{\circ}$ mês pós-operatório, nos grupos onde foram realizadas 2 incisões relaxantes de $8 \mathrm{~mm}$ (50\% do astigmatismo topográfico e $65 \%$ do refracio- nal) e 2 incisões de $6 \mathrm{~mm}$ (57\% do astigmatismo topográfico e $87 \%$ do refracional), mantendo-se sem alteração significante até o $12^{\circ}$ mês pós-operatório. No grupo de 1 incisão relaxante de $6 \mathrm{~mm}$, não foi alcançado redução significante do astigmatis-

\begin{tabular}{|c|c|c|c|c|c|}
\hline & \multirow{2}{*}{\multicolumn{5}{|c|}{ Astigmatismo }} \\
\hline & & & & & \\
\hline & № & ASTI & PRÉ-OP & 1ํMÉS & 12 MÊS \\
\hline \multirow{10}{*}{$2 \times 8 \mathrm{~mm}$} & \multirow[t]{2}{*}{01} & TOP & $2,40^{90}$ & $1,00^{96}$ & $1,00^{96}$ \\
\hline & & REF & $2,50^{90}$ & $1,00^{80}$ & $1,50^{105}$ \\
\hline & \multirow[t]{2}{*}{03} & TOP & $2,03^{108}$ & $1,67^{100}$ & $1,62^{100}$ \\
\hline & & REF & $2,25^{125}$ & $1,25^{109}$ & $0,75^{90}$ \\
\hline & \multirow[t]{2}{*}{04} & TOP & $2,52^{70}$ & $1,10^{70}$ & $0,75^{82}$ \\
\hline & & REF & $4,00^{70}$ & $1,00^{70}$ & 0 \\
\hline & \multirow[t]{2}{*}{08} & TOP & $1,84^{73}$ & $1,28^{78}$ & $1,50^{73}$ \\
\hline & & REF & $2,00^{90}$ & $1,00^{90}$ & $1,50^{80}$ \\
\hline & \multirow[t]{2}{*}{11} & TOP & $2,12^{96}$ & $0,46^{95}$ & $0,72^{107}$ \\
\hline & & REF & $2,00^{90}$ & $0,25^{80}$ & $0,50^{85}$ \\
\hline \multirow{12}{*}{$2 \times 6 \mathrm{~mm}$} & \multirow[t]{2}{*}{02} & TOP & $1,93^{61}$ & $1,56^{93}$ & $1,22^{92}$ \\
\hline & & REF & $2,00^{45}$ & 0 & $1,25^{90}$ \\
\hline & \multirow[t]{2}{*}{05} & TOP & $1,60^{90}$ & $1,30^{70}$ & $0,80^{71}$ \\
\hline & & REF & $2,00^{75}$ & 0 & 0 \\
\hline & \multirow[t]{2}{*}{07} & TOP & $1,82^{155}$ & $0,83^{158}$ & $1,06^{146}$ \\
\hline & & REF & $4,00^{180}$ & $0,50^{160}$ & $0,75^{140}$ \\
\hline & \multirow[t]{2}{*}{09} & TOP & $1,48^{90}$ & $0,40^{44}$ & $0,20^{45}$ \\
\hline & & REF & $1,00^{180}$ & $0,50^{90}$ & 0 \\
\hline & \multirow[t]{2}{*}{12} & TOP & $1,81^{160}$ & $0,24^{131}$ & $0,36^{139}$ \\
\hline & & REF & $1,50^{180}$ & 0 & $0,50^{175}$ \\
\hline & \multirow[t]{2}{*}{16} & TOP & $2,00^{140}$ & $0,26^{92}$ & $0,39^{124}$ \\
\hline & & REF & $1,75^{130}$ & $0,50^{100}$ & 0 \\
\hline \multirow{10}{*}{$1 \times 6 \mathrm{~mm}$} & \multirow[t]{2}{*}{06} & TOP & $1,00^{90}$ & $0,18^{17}$ & $1,25^{102}$ \\
\hline & & REF & $1,50^{90}$ & $1,00^{100}$ & $1,00^{100}$ \\
\hline & \multirow[t]{2}{*}{10} & TOP & $1,00^{75}$ & $1,20^{76}$ & $0,12^{15}$ \\
\hline & & REF & $1,00^{180}$ & $0,50^{90}$ & 0 \\
\hline & \multirow[t]{2}{*}{13} & TOP & $1,00^{71}$ & $1,41^{81}$ & $1,50^{74}$ \\
\hline & & REF & $1,50^{71}$ & $1,50^{90}$ & 0 \\
\hline & \multirow[t]{2}{*}{14} & TOP & $1,00^{90}$ & $0,75^{61}$ & $0,71^{90}$ \\
\hline & & REF & $1,00^{90}$ & $1,25^{99}$ & $0,75^{90}$ \\
\hline & \multirow[t]{2}{*}{15} & TOP & $1,30^{92}$ & $1,30^{95}$ & $1,58^{100}$ \\
\hline & & REF & $1,00^{100}$ & $1,00^{105}$ & $0,75^{90}$ \\
\hline
\end{tabular}

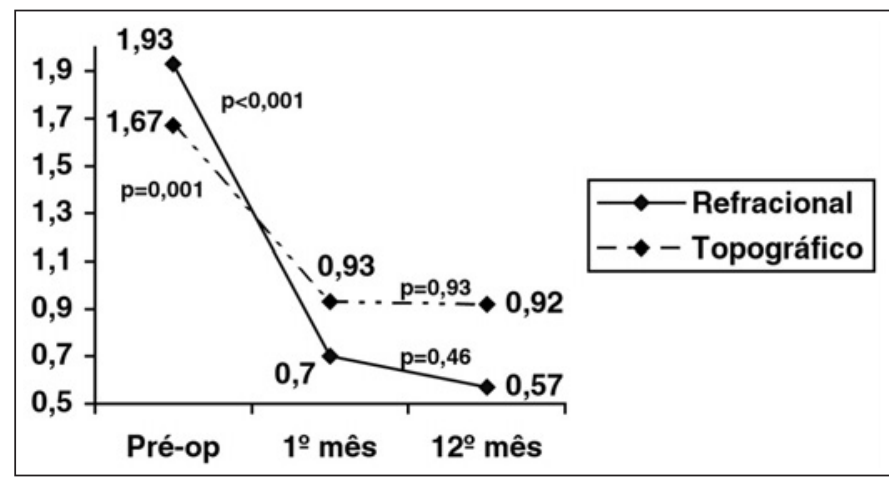

Gráfico 1 - Variação do valor médio do astigmatismo refracional e topográfico dos pacientes submetidos às incisões relaxantes limbares durante a cirurgia de catarata 


\begin{tabular}{|c|c|c|c|c|c|c|c|c|c|c|c|c|c|c|}
\hline \multirow[b]{3}{*}{ Grupo } & \multicolumn{14}{|c|}{ Astigmatismo } \\
\hline & \multicolumn{7}{|c|}{ Topográfico } & \multicolumn{7}{|c|}{ Refracional } \\
\hline & PRÉ & 19 & $12^{\circ}$ & RA & RV & p1 & p2 & PRÉ & $1^{\circ}$ & $12^{\circ}$ & RA & RV & p1 & p2 \\
\hline $2 \times 8 \mathrm{~mm}$ & 2,18 & 1,10 & 1,11 & 1,08 & 1,12 & 0,01 & 0,89 & 2,55 & 0,90 & 0,85 & 1,65 & 1,74 & 0,01 & 0,87 \\
\hline $2 \times 6 \mathrm{~mm}$ & 1,77 & 0,76 & 0,67 & 1,01 & 1,61 & $<0,01$ & 0,47 & 2,04 & 0,25 & 0,41 & 1,79 & 1,86 & $<0,01$ & 0,56 \\
\hline $1 \times 6 \mathrm{~mm}$ & 1,06 & 0,96 & 1,03 & 0,10 & 0,10 & 0,68 & 0,86 & 1,20 & 1,05 & 0,50 & 0,15 & 0,38 & 0,37 & 0,09 \\
\hline
\end{tabular}

mo pré-operatório topográfico e refracional no $1^{\circ}$ mês pósoperatório. No entanto, estes astigmatismos não apresentaram alteração significante também em relação ao $12^{\circ}$ mês pósoperatório (Tabela 2).

Não foi observado diferença significante dos valores médios, entre o pré-operatório e $12^{\circ}$ mês pós-operatório, dos eixos topográficos $\left(97^{\circ}\right.$ para $91^{\circ}$, respectivamente; $\left.p=0,29\right)$ e refracionais $\left(111^{\circ}\right.$ para $109^{\circ}$, respectivamente; $\left.\mathrm{p}=0,64\right)$ do astigmatismo. Não foi evidenciado, ainda, complicações pósoperatórias como "glare", aniseiconia, diplopia, desconforto, infecção da ferida e afinamentos ou ectasia corneana.

\section{DISCUSSÃO}

A busca da correção do astigmatismo, concomitante com a redução do grau esférico, durante a cirurgia de catarata, tem feito surgir várias técnicas ${ }^{(12-16)}$. Estas, no entanto, devem visar à diminuição do erro refrativo sem alterar as qualidades ópticas da córnea ${ }^{(6)}$, não levar ao surgimento de complicações e, ainda, alcançarem um efeito estável e duradouro.

As incisões relaxantes corneanas apresentam grande oscilação de seus resultados e flutuação pós-operatória freqüente na refração ${ }^{(16)}$, podendo levar ao aparecimento de complicações como diplopia, aniseiconia e alteração do senso espacial ${ }^{(12)}$.

As incisões relaxantes feitas no limbo durante a cirurgia de catarata, propostas por Gills, para correção de astigmatismo pré-operatório de até 3 dioptrias, têm-se mostrado eficazes na redução desta ametropia, com complicações pós-operatórias não-significantes ${ }^{(7,9)}$.

No presente estudo, foi alcançado uma redução significante do astigmatismo nos grupos $2 \times 6 \mathrm{~mm}$ (pacientes com 1 a 2 dioptrias de astigmatismo pré-operatório) e $2 \times 8 \mathrm{~mm}$ (pacientes com 2 a 3 dioptrias de astigmatismo pré-operatório) e esta redução manteve-se estável até o $12^{\circ}$ mês pós-operatório. Não foi observada a ocorrência de complicações pós-operatórias.

No grupo 1x6 mm (pacientes com 1 dioptria de astigmatismo pré-operatório), não houve redução significante do astigmatismo, no entanto, também não foi observado flutuação significante deste astigmatismo ao longo de 12 meses, mostrando que, mesmo que o objetivo não tenha sido atingindo, as IRL não levaram ao aumento desta ametropia, ectasia corneana ou outras complicações.

\section{CONCLUSÃO}

A realização de 2 incisões relaxantes de 6 e $8 \mathrm{~mm}$, para correção de astigmatismo pré-operatório de 1,1 a 2 dioptrias e 2,1 a 3 dioptrias, respectivamente, mostraram-se eficazes, seguras e com efeito estável ao longo do primeiro ano de acompanhamento. A realização de 1 incisão de $6 \mathrm{~mm}$ para redução de 1 dioptria de astigmatismo pré-operatório não se mostrou eficaz, no entanto, não levou a complicações pós-operatórias significativas.

\section{ABSTRACT}

Purpose: To evaluate astigmatism variation between preoperative, $1^{\text {st }}$ and $12^{\text {th }}$ postoperative month of patients who underwent cataract surgery with limbal relaxing incisions (LRI) aiming to reduce the preoperative astigmatism. Methods: Sixteen patients who underwent cataract surgery by the phacoemulsification technique with a $5.5 \mathrm{~mm}$ escleral incision, at the Altino Ventura Foudation, between April and July of 2002. The limbal relaxing incisions were performed according to Gills' modified nomogram (1D - 1 LRI of $6 \mathrm{~mm}$; 1-2D - 2 LRI of $6 \mathrm{~mm} ; 2-3 \mathrm{D}-2$ LRI of $8 \mathrm{~mm}$ ). They were done in the most curved meridians, determined by preoperative corneal topography. Results: Significant reduction in preoperative astigmatism was observed in the $1^{\text {st }}$ postoperative month in 2 limbal relaxing incisions of the $6 \mathrm{~mm}$ group (57\% topographic astigmatism and $87 \%$ refractional) and in 2 limbal relaxing incisions of the $8 \mathrm{~mm}$ group (50\% topographic astigmatism and $65 \%$ refractional), maintaining the reduction with no significant alteration until the $12^{\text {th }}$ postoperative month. The 1 limbal relaxing incision of the $6 \mathrm{~mm}$ group did not yield significant astigmatism reduction, but there was no significant alteration until de $12^{\text {th }}$ postoperative month. There were also no complications such as postoperative discomfort, glare, aniseiconia, diplopia, incision infection and corneal thinning or ectasia. Conclusion: Two limbal relaxing incisions of 8 and $6 \mathrm{~mm}$ aiming to correct preoperative astigmatism of 2 to $3 \mathrm{D}$ and 1 to $2 \mathrm{D}$, respectively, were safe and effective with a stable effect in the first postoperative follow-up year. The 1 limbal relaxing incision of $6 \mathrm{~mm}$ aiming to reduce 1 diopter of preoperative astigmatism was not effective, but it did not induce any significant postoperative complications. 
Keywords: Astigmatism/surgery; Cataract extraction; Limbus corneae/surgery; Corneal topography; Preoperative care; Postoperative care; Comparative study; Follow-up studies

\section{REFERÊNCIAS}

1. Bear JC, Richler A. Cylindrical refractive error: a population study in western Newfoundland. Am J Optom Physiol Opt. 1983;60(1):39-45.

2. Duke-Elder SS. Astigmatismo. In: Duke-Elder SS. Prática de refração em oftalmologia. 9a ed. Rio de Janeiro: Atheneu; 1984. p.57-61.

3. Barros EV, Dias VG. Incidência das ametropias no Hospital Universitário de Campo Grande entre 1986 e 1998. Arq Bras Oftalmol. 2000;63(3):203-8.

4. Moreira ATR. Astigmatismo. Arq Bras Oftalmol. 2001;64(3):271-2.

5. Schimiti RB, Costa VP, Gregui MJF, Kara-José N, Temporini ER. Prevalence of refractive errors and ocular disorders in preschool and schoolchildren of Ibiporã - PR, Brazil (1989-1996). Arq Bras Oftalmol. 2001;64(5):379-84.

6. Kohnen T, Koch DD. Methods to control astigmatism in cataract surgery. Curr Opin Ophthalmol 1996;7(1):75-80.

7. Cunha F, Arraes JC, Ribeiro MP, Almeida R, Cavalcanti R. Astigmatismo: correção cirúrgica durante a cirurgia de catarata. Mem Fund Altino Ventura. 2002;1:66-71.

8. Müller-Jensen K, Fischer P, Siepe U. Limbal relaxing incisions to correct astigmatism in clear corneal cataract surgery. J Refractive Surg. 1999;15(5):586-9.
9. Budak K, Friedman NJ, Koch DD. Limbal relaxing incisions with cataract surgery. J Cataract Refract Surg. 1998;24(4):503-8. Comment in: J Cataract Refract Surg. 2001;27(1):7-8.

10. Muller-Jensen K, Fischer P, Tan M. [Para-limbic relaxing incisions for reduction of astigmatism within the scope of cataract surgery] Klin Monatsbl Augenheilkd. 2000;217(5):257-62. German.

11. Waring III GO, Lynn MJ, Kutner MH. Stability of refraction after refractive keratotomy. In: Waring III GO, Lynn MJ, Kutner MH. Refractive keratotomy for myopia and astigmatism. St. Louis: Mosby; 1992. p.937-67.

12. Lindstrom RL, Agapitos PJ, Koch DD. Cataract surgery and astigmatic keratotomy. Int Ophthalmol Clin 1994;34(2):145-64.

13. Thornton SP. Inverse arcuate incision: a new approach to the correction of astigmatism. J Refract Corneal Surg. 1994;10(1):27-30. Comment in: J Refract Corneal Surg. 1994;10(4):465-6.

14. Price FW, Grene RB, Marks RG, Gonzales JS. Astigmatism reduction clinical trial: a multicenter prospective evaluation of the predictability of arcuate keratotomy. Evaluation of surgical nomogram predictability. ARC-T Study Group. Arch Ophthalmol. 1995;113(3):277-82. Erratum in: Arch Ophthalmol 1995; 113(5):577. Erratum in: Arch Ophthalmol 1995;113(5):577. Comment in: Arch Ophthalmol. 1995;113(10):1226.

15. Shimizu K, Misawa A, Suzuki Y. Toric intraocular lens: correcting astigmatism while controlling axis shift. J Cataract Refract Surg. 1994;20(5):523-6.

16. Buzard KA, Laranjeira E, Fundingsland BR. Clinical results of arcuate incisions to correct astigmatism. J Cataract Refract Surg. 1996;22(8):1062-9.

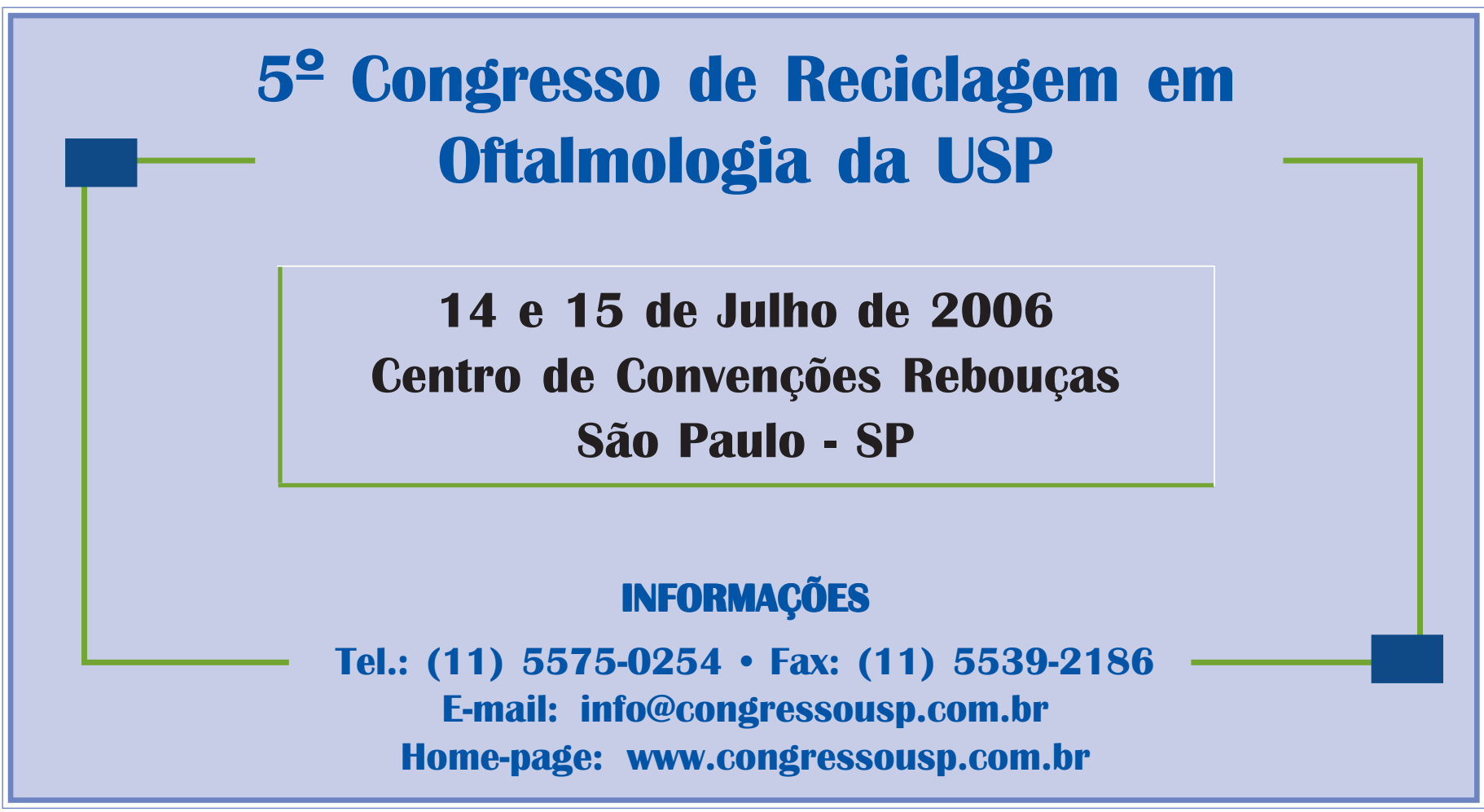

